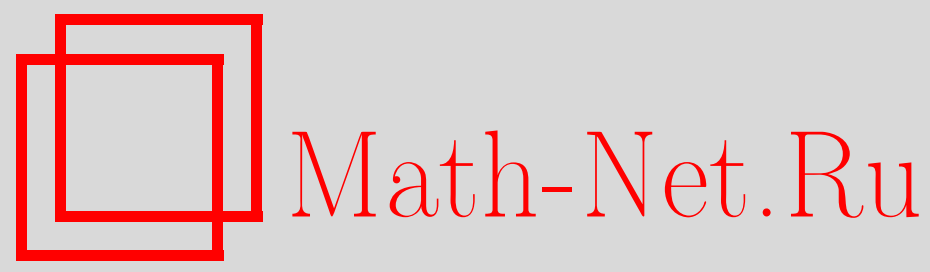

В. А. Кощеев, О разбиении компактов, допускающих $T$ системы комплексных функций, Матем. заметки, 1997, том 62, выпуск 2, 259-267

DOI: https://doi.org/10.4213/mzm1609

Использование Общероссийского математического портала Math-Net.Ru подразумевает, что вы прочитали и согласны с пользовательским соглашением http://www . mathnet.ru/rus/agreement

Параметры загрузки:

IP: 54.84 .234 .179

26 апреля 2023 г., $17: 23: 21$

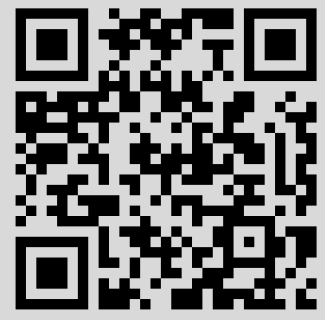




\section{О РАЗБИЕНИИ КОМПАКТОВ, ДОПУСКАЮЩИХ Т-СИСТЕМЫ КОМПЛЕКСНЫХ ФУНКЦИЙ}

\section{В. А. Кощеев}

Рассматриваются полунепрерывные сверху разбиения на континуумы компакта, допускающего чебышевскую систему непрерьвных комплексных функций. В частности, для пространства разбиения Мура доказывается возможность вложения в двумерную сферу его циклических элементов.

Библиография: 8 названий.

Введение. Рассматриваются компакты $Q$ (т.е. метризуемые бикомпактные пространства), для которых в пространстве $C(Q, \mathbb{C})$ непрерьвных комплексных функций существует система Чебышева порядка $n($ кратко $T(n, \mathbb{C})$-система или просто $T$-система)

$$
\left\{f_{1}(q), \ldots, f_{n}(q)\right\}
$$

т.е. система линейно независимых функций, полиномы по которой с комплексными коэффициентами обладают свойством единственности элемента наилучшего приближения для любой функции из $C(Q, \mathbb{C})$.

$T$-системы комплексных функций изучались многими авторами [1]-[4]. В 1948 году А. Н. Колмогоровым получен критерий для $T(n, \mathbb{C})$-систем [1]. В работах Шенберга и Янга [2] и Овердека [3] показано, что если $Q$ - двумерньй или одномерньй локально связньй компакт и на $Q$ существует $T(n, \mathbb{C})$-система порядка $n>1$, то $Q$ гомеоморфен подмножеству плоскости $\mathbb{C}$.

В работе рассматриваются полунепрерьвные сверху разбиения на континуумы компакта $Q$, допускающего $T(n, \mathbb{C})$-систему $(1)$ порядка $n>2$. В частности, полунепрерьвньм сверху является разбиение Мура $\mathscr{D}$ компакта $Q$ на компоненты множества $\bar{N}$ и отдельные точки дополнения $Q \backslash \bar{N}$, где $N$ - множество точек, в которых $Q$ не является локально связным. В п. 2 доказывается возможность вложения в сферу $S^{2}$ циклических элементов пространства разбиения $\mathscr{D}$. В п. 1 для метрического континуума $Q$ и натурального $n$ рассматривается семейство $e_{n} Q$ всех конечных подмножеств из $Q$ мощности точно равной $n$ и доказьвается связность этого семейства в хаусдорфовой метрике. Результаты п. 1 используются в п. 2 .

Пункт 2 работы выполнен при финансовой поддержке Российского фонда фундаментальных исследований, грант № 93-011-196. 
1. Связность $e_{n} Q$. Подчеркнем отличие определенного во введении семейства $e_{n} Q$ от часто встречающегося в математической литературе семейства $\exp _{n} Q$ всех конечных подмножеств пространства $Q$ мощности, не превосходящей $n$ (см., например, [5]), связность которого хорошо известна [6, теорема 12, с. 147].

Обозначим через $d(\cdot, \cdot)$ хаусдорфову метрику в пространстве $\exp Q$ всех непустых замкнутых подмножеств компакта $Q$. Семейство $e_{n} Q$ с индуцированной метрикой обозначим тем же символом.

ЛЕмма 1. Для метрического континуума $Q$ пространство $e_{n} Q$ связно при любом натуральном $n$.

ДокАЗАТЕЛЬСТво. Для $n=1$ утверждение очевидно. Предположим по индукции, что для любого $m \leqslant n$ и любого метрического континуума $Q e_{m} Q$ связно, и докажем связность $e_{n+1} Q$.

По теореме Мазуркевича и Янишевского [6, теорема 1, с. 200] для любых двух точек из $Q$ существует континуум, неприводимьй между этими точками. Пусть $a, b \in Q$ $(a \neq b)$ и континуум $K \subset Q$ неприводим между $a$ и $b$, т.е. $a, b \in K$, но эти точки нельзя соединить никаким собственным подконтинуумом континуума $K$. Докажем вначале связность $e_{n+1} K$.

По теореме Борсука и Мазуркевича [6, теорема 3, с. 195] существует монотонное семейство континуумов из $K$, образуюшее в пространстве $\exp K$ дугу с концами $a$ и $K$, т.е. существует гомеоморфизм

$$
F:[0,1] \rightarrow F([0,1]) \subset \exp K, \quad F(0)=a, \quad F(1)=K,
$$

такой, что для любого $t \in[0,1] F(t)$ - континуум из $K$, из $t<t^{\prime}$ следует $F(t) \subset F\left(t^{\prime}\right)$. Аналогично, существует гомеоморфизм

$$
G:[0,1] \rightarrow G([0,1]) \subset \exp K, \quad G(0)=K, \quad G(1)=b,
$$

причем для любого $\tau \in[0,1] G(\tau)$ - континуум из $K$, из $\tau>\tau^{\prime}$ следует $G(\tau) \subset G\left(\tau^{\prime}\right)$. Заметим, что ввиду неприводимости $K$ между точками $a$ и $b$ вьполнено $b \notin F(t)$ для любого $t \in[0,1)$.

Пару чисел $(t, \tau) \in[0,1) \times(0,1]$ назовем допустимой, если $F(t) \cap G(\tau)=\varnothing$. Для допустимой пары $(t, \tau)$ рассмотрим множество

$$
M_{t, \tau}=\left\{\left\{q_{1}, q_{2}, \ldots, q_{n}, p\right\} \in e_{n+1} K:\left\{q_{1}, \ldots, q_{n}\right\} \in e_{n} F(t), p \in G(\tau)\right\} .
$$

Так как $e_{n} F(t)$ связно по предположению индукции и $M_{t, \tau}$ гомеоморфно произведению $e_{n} F(t) \times G(\tau)$, то $M_{t, \tau}$ связно.

Для любых допустимых пар $(t, \tau)$ и $\left(t^{\prime}, \tau^{\prime}\right)$

$$
M_{t, \tau} \cap M_{t^{\prime}, \tau^{\prime}} \neq \varnothing
$$

В самом деле, с одной стороны, в силу монотонности семейства $G(\cdot) b \in G(\tau) \cap G\left(\tau^{\prime}\right)$. С другой стороны, ввиду монотонности семейства $F(\cdot)$ либо $F(t) \subset F\left(t^{\prime}\right)$, либо $F\left(t^{\prime}\right) \subset F(t)$.

Из доказанного следует, что объединение $M$ множеств $M_{t, \tau}$ по всем допустимым парам $(t, \tau)$ является связным множеством в $e_{n+1} K$. 
Пусть $\left\{q_{1}, \ldots, q_{n}, q_{n+1}\right\} \in e_{n+1} K$. Рассмотрим вначале случай $q_{n+1}=b$. Так как $F(1)=K$, объединение континуумов $F(t)$ по всем $t \in[0,1)$ плотно в $K$, поэтому для любого $\varepsilon>0$ существует число $t \in(0,1)$ такое, что

$$
\max _{i=1, \ldots, n} \rho\left(q_{i}, F(t)\right)<\varepsilon
$$

где $\rho(\cdot, \cdot)$ - метрика в $K$. Откуда следует, что найдется такой элемент $\left\{q_{1}^{\prime}, \ldots, q_{n}^{\prime}\right\} \in$ $e_{n} F(t)$, что $\rho\left(q_{i}, q_{i}^{\prime}\right)<\varepsilon(i=1, \ldots, n)$, следовательно, $\left\{q_{1}, \ldots, q_{n}, b\right\} \in \bar{M}$, где замыкание берется в $e_{n+1} K$. Пусть теперь $\left\{q_{1}, \ldots, q_{n}, q_{n+1}\right\} \in e_{n+1}(K \backslash b)$,

$$
\tau_{0}=\inf _{\tau \in[0,1]}\left\{q_{i} \notin G(\tau), i=1, \ldots, n+1\right\}
$$

Очевидно, что $G\left(\tau_{0}\right) \cap\left\{q_{1}, \ldots, q_{n}, q_{n+1}\right\} \neq \varnothing$. Перенумеровав точки $q_{i}$, если это необходимо, можно считать, что $q_{n+1} \in G\left(\tau_{0}\right)$. Тогда для любого $\varepsilon>0$ найдется $\tau>\tau_{0}$ такое, что $\rho\left(q_{n+1}, G(\tau)\right)<\varepsilon$, поэтому $\left\{q_{1}, \ldots, q_{n+1}\right\}$ принадлежит замыканию в $e_{n+1} K$ связного множества

$$
\bigcup_{\tau>\tau_{0}}\left\{\left\{q_{1}, \ldots, q_{n}, q\right\}: q \in G(\tau)\right\}
$$

которое, как только что было доказано, пересекается с $\bar{M}$.

Итак, каждая точка из $e_{n+1} K$ либо принадлежит связному множеству $\bar{M}$, либо содержится в замыкании некоторого связного множества, пересечение которого с $\bar{M}$ непусто. Значит, любые две точки из $e_{n+1} K$ можно соединить связным подмножеством, откуда следует связность $e_{n+1} K$.

Если $A_{1}, A_{2}$ - два подконтинуума из $Q, A_{1} \cap A_{2} \neq \varnothing$, и для любого $m \leqslant n+1$ $e_{m} A_{i}$ связно $(i=1,2)$, то, как нетрудно показать, $e_{m}\left(A_{1} \cup A_{2}\right)$ также связно для тех же $m$. Аналогичное утверждение верно и для любого конечного числа подконтинуумов, пересечение которых непусто.

Пусть $\left\{q_{1}, \ldots, q_{n+1}\right\} \in e_{n+1} Q,\left\{q_{1}^{\prime}, \ldots, q_{n+1}^{\prime}\right\} \in e_{n+1} Q, q_{0} \in Q ; K_{i}, K_{i}^{\prime}$ - подконтинуумы из $Q$, неприводимые между $q_{0}$ и $q_{i}$ соответственно между $q_{0}$ и $q_{i}^{\prime}, i=1, \ldots, n+1$. В силу доказанного множество

$$
e_{n+1}\left(\bigcup_{i=1}^{n+1} K_{i} \cup K_{i}^{\prime}\right)
$$

связно. Таким образом, любые два элемента из $e_{n+1} Q$ можно соединить связным подмножеством, следовательно, $e_{n+1} Q$ связно.

2. Разбиения $Q$. Как известно, разбиением пространства $Q$ назьвается семейство $\mathscr{D}$ замкнутых непустых и непересекающихся подмножеств из $Q$, объединение которых представляет собой все пространство $Q$. Семейство $\mathscr{D}$ в своей фактортопологии называется пространством разбиения, отображение $P: Q \rightarrow \mathscr{D}$, называемое проекиией, определяется условием $P(q)=D$ тогда и только тогда, когда $q \in D \in \mathscr{D}$. Разбиение $\mathscr{D}$ назьвается полунепрерывным сверху, если для всякого открытого множества $G \subset Q$ объединение всех $D \in \mathscr{D}$, содержащихся в $G$, открыто; это эквивалентно условию, что $P$ - замкнутое отображение [6, с. 72$]$. 
ТЕОРема 1 [6, с. 74]. Каждое из следующих условий является необходимым и достаточным для того, чтобы разбиение $\mathscr{D}$ компакта $Q$ было полунепрерывным сверху:

1) из условия $D \cap \operatorname{Li} D_{n} \neq \varnothing$ следует, что Ls $D_{n} \subset D$, әде $D, D_{n} \in \mathscr{D}$;

2) если последовательность $D_{1}, D_{2}, \ldots$ сходится, то ее предел содержится в единственном әлементе разбиения $\mathscr{D}$.

По теореме П. С. Александрова [6, теорема 1, с. 73] полунепрерьвное сверху разбиение компакта (в своей фактортопологии) гомеоморфно компактному метрическому пространству.

Tеорема 2 (Мур, [6, с. 253]). Пусть $Q-$ континуум и $N$ - множество точек, в которых $Q$ не является локально связным. Тогда пространство разбиения $Q$ на компоненты множества $\bar{N}$ и отдельные точки дополнения $Q \backslash \bar{N}$ является локально связным континуумом.

Другими словами, существует непрерьвное отображение $f$ компакта $Q$ на $Y$ такое, что $Y$ есть локально связньй континуум, и семейство множеств $f^{-1}(y), y \in Y$, совпадает с семейством компонент множества $\bar{N}$ и отдельных точек дополнения $Q \backslash \bar{N}$.

Заметим, что отображение $f$ является монотонным, т.е. прообразы связных множеств связны $[6$, теорема 9 , с. 140$]$.

Разбиения, вообще говоря, отличные от указанных разбиений Мура, возникают при рассмотрении замкнутых подмножеств пространства разбиения и их полных прообразов при факторотображении. Точнее, пусть $\mathscr{D}$ - разбиение Мура компакта $Q$ с пространством разбиения $Y$ и проекцией $f$. Для замкнутого подмножества $Y_{0} \subset Y$ рассмотрим разбиение множества $f^{-1}\left(Y_{0}\right)$, индуцированное разбиением $\mathscr{D}$, на семейство замкнутых связных подмножеств $\left\{f^{-1}(y): y \in Y_{0}\right\}$. Разбиение, индуцированное разбиением Мура (также как и само разбиение Мура), является полунепрерывным сверху. Более того, разбиение любого компактногопространства на компонентыполунепрерывно сверху $[6$, теорема 1 , с. 190]. Отметим также, что если $f$ - непрерьвное отображение компакта $X$ на компакт $Y$, то разбиение $X=\bigcup_{y \in Y}\left\{f^{-1}(y)\right\}$ полунепрерывно сверху [6, теоремa 3, с. 73].

Пусть даны компакт $Q \subset S^{2}$ и его полунепрерьвное сверху разбиение $\mathscr{D}$ на континуумы $D \in \mathscr{D}$ с пространством разбиения $Y$ и проекцией $f$. Рассмотрим компакт $Q^{\prime} \supset Q$ (называемьй далее ассоциированным с $Q$ ) и его разбиение $\mathscr{D}^{\prime}$, которые связаны с $Q$ и разбиением $\mathscr{D}$ следующим образом. Если элемент разбиения $D \in \mathscr{D}$ не разделяет сферу $S^{2}$, то полагаем $D=D^{\prime} \in \mathscr{D}^{\prime}$. Пусть $D \in \mathscr{D}$ разделяет $S^{2}$. Обозначим через $D^{\prime}$ множество из $S^{2}$, которое получается из $D$ добавлением к нему всех тех компонент дополнения $S^{2} \backslash D$, которые не содержат точек множества $Q$. Так как компоненты открытого множества $S^{2} \backslash D$ открыты, то $D^{\prime}$ замкнуто. Положим $Q^{\prime}=\cup D^{\prime}$, где объединение берется по всем $D^{\prime} \in \mathscr{D}^{\prime}$. Подмножество $Q^{\prime} \subset S^{2}$ замкнуто, и $\mathscr{D}^{\prime}$ является его полунепрерьвньм сверху разбиением на континуумы $D^{\prime} \in \mathscr{D}^{\prime}$. В самом деле, если $x \in \operatorname{Li} D_{n}^{\prime}$ и $x \notin \cup D_{n}^{\prime}$, то $x \in \operatorname{Li} D_{n} \subset Q \subset Q^{\prime}$, так как расстояния от $x$ до множеств $D_{n}^{\prime}$ и $D_{n}$ совпадают. Далее, пусть $D^{\prime}, F^{\prime} \in \mathscr{D}^{\prime}$ и $D^{\prime} \neq F^{\prime}$. Тогда $D^{\prime} \cap F^{\prime}=\varnothing$. Действительно, если $D^{\prime}=D \in \mathscr{D}$ или $F^{\prime}=F \in \mathscr{D}$, то утверждение следует из определения семейства $\mathscr{D}^{\prime}$. Пусть $D^{\prime} \backslash D \neq \varnothing$ и $F^{\prime} \backslash F \neq \varnothing$. Предположим, что $x \in D^{\prime} \cap F^{\prime}$. Обозначим через $A(B)$ компоненту точки $x$ в множестве $S^{2} \backslash D\left(S^{2} \backslash F\right.$ соответственно). Так как $\bar{B} \cap F \neq \varnothing$, а $x$ и $F$ лежат в разных компонентах множества $S^{2} \backslash D$, то $B \cap D \neq \varnothing$, что 
противоречит определению семейства $\mathscr{D}^{\prime}$. Проверим полунепрерывность сверху разбиения $\mathscr{D}^{\prime}$. В силу теоремы 1 достаточно показать, что $D^{\prime} \cap \operatorname{Li} D_{n}^{\prime} \neq \varnothing$ влечет Ls $D_{n}^{\prime} \subset D^{\prime}$. Пусть $x \in D^{\prime} \cap \operatorname{Li} D_{n}^{\prime}$. Тогда $x \in D \cap \operatorname{Li} D_{n}$ и по теореме 1 Ls $D_{n}^{\prime}=\operatorname{Ls} D_{n} \subset D \subset D^{\prime}$. Наконец, связность $D^{\prime} \in \mathscr{D}^{\prime}$ следует из связности $D$ и того факта, что пересечение $D$ с замьканием компоненты множества $S^{2} \backslash D$ непусто.

Рассмотрим отображение $\mathscr{F}: Q^{\prime} \rightarrow Y$, полагая $\mathscr{F}\left(q^{\prime}\right)=f(D)$ для $q^{\prime} \in D^{\prime}$. Непрерывность $\mathscr{F}$ легко проверяется: если $q_{n}^{\prime} \rightarrow q^{\prime}, q_{n}^{\prime} \in D_{n}^{\prime}, q^{\prime} \in D^{\prime}$, то $q^{\prime} \in D$ и найдется такая последовательность точек $q_{n} \in D_{n}$ (за исключением случая стационарной последовательности $\left.\left\{D_{n}^{\prime}\right\}\right)$, что $q_{n} \rightarrow q^{\prime}$, откуда $\mathscr{F}\left(q_{n}^{\prime}\right)=f\left(q_{n}\right) \rightarrow f\left(q^{\prime}\right)=\mathscr{F}\left(q^{\prime}\right)$. Монотонность $\mathscr{F}$ следует из связности элементов разбиения $\mathscr{D}^{\prime}$ [6, теорема 9, с. 140$]$.

Лемма 2. Компакт $Q^{\prime}$, ассоциированный с континуумом $Q \subset S^{2}$, пространством разбиения которого является $\Theta$-кривая $Y$, состоящая из трех дуг $l_{0}, l_{1}, l_{2}$ (каждая пара которых имеет общими только кониевые точки), разделяет сферу $S^{2}$ на три области $\Omega^{0}, \Omega^{1}$ и $\Omega^{2}$ - компоненты множества $S^{2} \backslash Q^{\prime}=\bigcup_{j=0}^{2} \Omega^{j}$. $\Pi р и$ этом $\operatorname{Fr} \Omega^{j} \subset f^{-1}\left(l_{j} \cup l_{j-1}\right)($ индексы приведены по $\bmod 3)$.

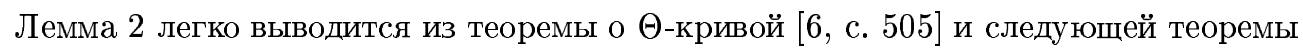
Мура [6, теорема 8, с. 526].

Теорема 3 (Мур). Пространство полунепрерывного сверху разбиения сферы $S^{2}$ на континуумы, не разделяющие $S^{2}$, гомеоморфно $S^{2}$.

Другими словами, если $g$ - непрерьвное отображение сферы $S^{2}$ на компакт $Z$ такое, что для каждого $z \in Z$ множество $g^{-1}(z)$ - континуум, не разделяющий $S^{2}$, то $Z$ гомеоморфин $S^{2}$.

Для получения разбиения сферы $S^{2}$, о котором идет речь в теореме 3 , из разбиения $\mathscr{D}^{\prime}$ компакта $Q^{\prime} \subset S^{2}$ семейство $\mathscr{D}^{\prime}$ следует дополнить одноэлементными множествами из $S^{2} \backslash Q^{\prime}$. Отметим также, что в условиях леммы 2 элементы разбиения $\mathscr{D}^{\prime}$ не разделяют $S^{2}$. В самом деле, пусть $D \in \mathscr{D}$ разделяет $S^{2}$ и $f(D)=y \in Y$. Так как $Y \backslash y$ связно и $f$ - монотонное отображение, $Q \backslash D=f^{-1}(Y \backslash y)$ лежит в одной компоненте множества $S^{2} \backslash D$, скажем в компоненте $G$, поэтому при переходе от $D$ к $D^{\prime}$ все остальные компоненты множества $S^{2} \backslash D$ “закрашиваются”, следовательно, $S^{2} \backslash D^{\prime}=G$ связно.

Лемма 3. Пусть на компакте $Q$ задана $T(n, \mathbb{C})$-система (1) порядка $n>2, G$ открытое подмножсество из $Q$ такое, что $e_{n-2} G \neq \varnothing, e_{2}(Q \backslash G) \neq \varnothing$. Тогда для любого әлемента $\mathscr{P} \in e_{n-2} G$ существует непрерывное и инвективное отобрахенuе

$$
\xi(\mathscr{P}, \cdot): Q \backslash \mathscr{P} \rightarrow \mathbb{C P}^{1}
$$

такое, что отображсение

$$
\xi: e_{n-2} G \rightarrow C\left(Q \backslash G, \mathbb{C P}^{1}\right)
$$

непрерывно, где

$$
\xi(\mathscr{P})=\left.\xi(\mathscr{P}, \cdot)\right|_{Q \backslash G}: Q \backslash G \rightarrow \mathbb{C P}^{1}
$$

есть сужение отображения $\xi(\mathscr{P}, \cdot)$ на множсество $Q \backslash G$. 
ДокАЗАТЕЛЬСтво. Пусть $\mathscr{P}=\left\{p_{1}, \ldots, p_{n-2}\right\} \in e_{n-2} Q, n>2$, и система функций $(1)$ является $T$-системой из $C(Q, \mathbb{C})$. Обозначим через $F(\mathscr{P})(n-2) \times n$-матрицу $\left(a_{i j}\right)$, полагая $a_{i j}=f_{j}\left(p_{i}\right)$. Для $q \in Q$ матрица $F(\mathscr{P}, q)$ получается из $F(\mathscr{P})$ добавлением $(n-1)$-й строки вида $\left(f_{1}(q), \ldots, f_{n}(q)\right)$. Определитель $(n-1)$-го порядка матрицы $F(\mathscr{P}, q)$, полученньй вычеркиванием $i$-го столбца, обозначим $\Delta_{i}(\mathscr{P}, q)$.

Для любого $\mathscr{P} \in e_{n-2} Q$ существует вектор $l=\left(l_{1}, l_{2}\right)$ с целыми положительными координатами $1 \leqslant l_{1}<l_{2} \leqslant n$ такой, что отображение

$$
q \rightarrow \xi_{l}(\mathscr{P}, q)=\left(\Delta_{l_{1}}(\mathscr{P}, q): \Delta_{l_{2}}(\mathscr{P}, q)\right) \in \mathbb{C P}^{1}
$$

определенное на множестве $Q \backslash \mathscr{P}$, непрерывно и инъективно [4, лемма 1]. Вектор $l$, вообще говоря, зависит от элемента $\mathscr{P}$. Однако, если задано открытое множество $G \subset Q$ и вьполнены условия леммы 3 , то вектор $l$ может быть выбран одинаковым для всех $\mathscr{P} \in e_{n-2} G$. Для этого зафиксируем $\left\{q_{1}, q_{2}\right\} \in e_{2}(Q \backslash G)$ и от системы (1) перейдем к другой $T(n, \mathbb{C})$-системе на $Q$

$$
\left\{\varphi_{1}(q), \ldots, \varphi_{n}(q)\right\}
$$

составленной из полиномов по системе (1) таких, что $\varphi_{i}\left(q_{j}\right)=0$ для $i=1, \ldots, n-2$, $j=1,2$. Это можно сделать в силу критерия Колмогорова для $T(n, \mathbb{C})$-систем $[1]$ (см. также [4]).

Для системы $\left\{\varphi_{i}(q)\right\}$ составим матрицу $\Phi(\mathscr{P}, q)$ по аналогии с матрищей $F(\mathscr{P}, q)$ для $(1)$ и определители $\Delta_{n-1}(\mathscr{P}, q)$ и $\Delta_{n}(\mathscr{P}, q)$. Тогда вектор $l_{0}=(n-1, n)$ задает требуемое отображение

$$
\xi=\xi_{l_{0}}: \mathscr{P} \rightarrow \xi_{l_{0}}(\mathscr{P}, \cdot) \in C\left(Q \backslash G, \mathbb{C P}^{1}\right)
$$

Обозначим через $K_{1}$ граф “три домика, три колодца”, через $K_{2}$ полньй граф с пятью вершинами.

Теорема 4. Если на континууме $Q$ существует $T(n, \mathbb{C})$-система (1) порядка $n>2$, то пространство полунепрерывного сверху разбиения $Q$ на континуумы не содерэит топологически графов $K_{1} u K_{2}$.

ДокАЗАтЕльство. Рассмотрим геометрическую реализацию графа $K_{1}$ в $\mathbb{R}^{3}$. A именно, рассмотрим граф, образованньй вершинами тетраэдра $A B C D$, ребрами $A B$, $A C, B D, C D$, вершинами $E$ и $F$ - серединами отрезков $[A, D]$ и $[B, C]$ соответственно и ребрами $E F, A E, E D, B F$ и $F C$.

Пусть $\mathscr{D}$ - полунепрерьвное сверху разбиение $Q$ на континуумы, $Y$ - пространство разбиения $\mathscr{D}, f: Q \rightarrow Y$ - проекция. Если $Y$ содержит (топологически) $K_{1}$ в качестве собственного подмножества, то система (1) является $T$-системой на континууме $f^{-1}\left(K_{1}\right)$ с индуцированным разбиением, поэтому доказательство сводится к случаю, когда $Y=K_{1}$ (с точностью до гомеоморфизма).

Введем в рассмотрение точки $L \in(A, E), M \in(E, D), N^{\prime} \in(E, F)$ и положим

$$
G=[E, L) \cup[E, M) \cup\left[E, N^{\prime}\right) .
$$

Пусть $K$ - подграф графа $K_{1}$, порожденный вершинами $A, B, C, D$ и $F, Q_{K}=f^{-1}(K)$. Разбиение континуума $Q_{K}$, индуцированное разбиением $\mathscr{D}$, и соответствующую проекцию на факторпространство $K$ обозначим теми же символами $\mathscr{D}$ и $f$. 
Рассмотрим триод

$$
T=\left[L^{\prime}, E\right] \cup\left[M^{\prime}, E\right] \cup\left[N^{\prime \prime}, E\right] \subset G,
$$

где $L^{\prime} \in(L, E), M^{\prime} \in(M, E), N^{\prime \prime} \in\left(N^{\prime}, E\right)$. Для любого элемента $\mathscr{P} \in e_{n-2} f^{-1}(T)$ рассмотрим в соответствии с леммой 3 вложение $\xi(\mathscr{P}, \cdot)$ компакта $Q_{K}$ в $S^{2}$ и положим $\xi\left(\mathscr{P}, Q_{K}\right)=Q_{K, \mathscr{P}}$. От компакта $Q_{K, \mathscr{P}} \subset S^{2}$ перейдем к ассоциированному с ним компакту $Q_{K, \mathscr{P}}^{\prime}$ с тем же пространством разбиения $K$ и проекцией $\mathscr{F}=\mathscr{F} \mathscr{P}$. Из определения ассоциированного компакта следует, что $\xi\left(\left(\mathscr{P}, f^{-1}(y)\right) \neq \mathscr{F}^{-1}(y)\right.$ для $y \in K$ только в том случае, если $\xi\left(\mathscr{P}, f^{-1}(y)\right)$ разделяет $S^{2}$ и среди компонент множества $S^{2} \backslash \xi\left(\mathscr{P}, f^{-1}(y)\right)$ имеются такие, которые не содержат точек множества $\xi\left(\mathscr{P}, f^{-1}(K)\right)$.

Покажем, что если $y \in K$ и $\xi\left(\mathscr{P}, f^{-1}(y)\right) \neq \mathscr{F}^{-1}(y)$, то

$$
\mathscr{F}^{-1}(y) \cap \xi\left(\mathscr{P}, f^{-1}(Y \backslash(K \cup G))\right)=\varnothing .
$$

Предположим противное. Пусть $y \in K, \xi\left(\mathscr{P}, f^{-1}(y)\right)$ разделяет $S^{2}$ и $H_{\mathscr{P}}$ - одна из компонент множества $S^{2} \backslash \xi\left(\mathscr{P}, f^{-1}(y)\right)$, которая “закрашивается" при переходе к ассоциированному компакту $Q_{K, \mathscr{P}}^{\prime}$, причем

$$
H_{\mathscr{P}} \cap \xi\left(\mathscr{P}, f^{-1}(Y \backslash(K \cup G))\right) \neq \varnothing .
$$

Пусть, например, $H_{\mathscr{P}}$ содержит точки множества $\xi\left(\mathscr{P}, f^{-1}(D, M]\right)$. В силу инъективности $\xi(\mathscr{P}, \cdot)$ (по лемме 3$)$ и монотонности $f \xi\left(\mathscr{P}, f^{-1}(D, M]\right) \subset H_{\mathscr{P}}$. По определению $Q_{K, \mathscr{P}}^{\prime} \xi\left(\mathscr{P}, f^{-1}(K \backslash y)\right) \cap H_{\mathscr{P}}=\varnothing$. Пусть $q \in f^{-1}(K \backslash y), p \in f^{-1}(M)$, тогда $\xi(\mathscr{P}, q) \notin \bar{H} \mathscr{P}, \xi(\mathscr{P}, p) \in H_{\mathscr{P}}$. Разделитель Fr $H_{\mathscr{P}}$ содержит замкнутьй неприводимьй разделитель $R_{\mathscr{P}} \subset \operatorname{Fr} H \mathscr{P} \subset \xi\left(\mathscr{P}, f^{-1}(y)\right)$ между точками $\xi(\mathscr{P}, q)$ и $\xi(\mathscr{P}, p)$ $\left[6\right.$, теорема 3 , с. 250], причем $R_{\mathscr{P}}$ - общая граница двух компонент множества $S^{2} \backslash R_{\mathscr{P}}$ $\left[6\right.$, теорема 2 , с. 469], одна из которых, скажем $A_{\mathscr{P}}$, содержит

$$
H_{\mathscr{P}} \supset \xi\left(\mathscr{P}, f^{-1}(D, M]\right) \ni \xi(\mathscr{P}, p),
$$

а другая $B \mathscr{P}$ содержит

$$
\xi\left(\mathscr{P}, f^{-1}(K \backslash y)\right) \ni \xi(\mathscr{P}, q) .
$$

Понятие замкнутого неприводимого разделителя сферы $S^{2}$ является топологическим инвариантом [6, с. 470] (см. также [7]), поэтому для любого $\mathscr{P}^{\prime} \in e_{n-2} f^{-1}(T)$ множество $R_{\mathscr{P} \prime}=\xi\left(\mathscr{P}^{\prime}, R\right)$, где $R=\xi(\mathscr{P})^{-1}(R \mathscr{P})$, также является замкнутым неприводимым разделителем $S^{2}$, причем $S^{2} \backslash R_{\mathscr{P}}$ - объединение двух компонент $A_{\mathscr{P} \prime}$ и $B_{\mathscr{P} \prime^{\prime}}[6$, теорема 2, с. 469; теорема 7, с. 490].

Покажем, что для любого $\mathscr{P}^{\prime} \in e_{n-2} f^{-1}(T)$ связные множества

$$
\xi\left(\mathscr{P}^{\prime}, f^{-1}(D, M]\right) \ni \xi\left(\mathscr{P}^{\prime}, p\right), \quad \xi\left(\mathscr{P}^{\prime}, f^{-1}(K \backslash y)\right) \ni \xi\left(\mathscr{P}^{\prime}, q\right)
$$

принадлежат разным компонентам. Для этого рассмотрим подмножество $U$ элементов $\mathscr{P}^{\prime} \in e_{n-2} f^{-1}(T)$, для которых точки $\xi\left(\mathscr{P}^{\prime}, p\right)$ и $\xi\left(\mathscr{P}^{\prime}, q\right)$ лежат в разных компонентах дополнения $S^{2} \backslash R_{\mathscr{P}^{\prime}}$, и подмножество $V=\left[e_{n-2} f^{-1}(T)\right] \backslash U$. Нетрудно заметить, что множество $V$ открыто.

Покажем, что $U$ также открыто. Пусть $\mathscr{P}_{0} \in U$. Рассмотрим на $S^{2}$ два открытых диска $V_{p} \ni \xi\left(\mathscr{P}_{0}, p\right)$ и $V_{q} \ni \xi\left(\mathscr{P}_{0}, q\right)$, замькания которых не пересекаются ни между 
собой, ни с множеством $R_{\mathscr{P}_{0}}$. Существует число $\varepsilon(0<\varepsilon<2)$ такое, что для любого $q^{\prime} \in \mathbb{R}$ геометрически правильньй диск с центром в $\xi\left(\mathscr{P}_{0}, q^{\prime}\right)$ и радиусом $\varepsilon$ содержится в кольце $S^{2} \backslash\left(V_{p} \cup V_{q}\right)$. Возьмем в $e_{n-2} f^{-1}(T)$ столь малую окрестность $W$ элемента $\mathscr{P}_{0}$, чтобы вьполнялись условия: для любого $\mathscr{P}^{\prime} \in W \quad \xi\left(\mathscr{P}^{\prime}, p\right) \in V_{p}, \xi\left(\mathscr{P}^{\prime}, q\right) \in V_{q}$; $R_{\mathscr{P} \prime} \subset S^{2} \backslash\left(\bar{V}_{p} \cup \bar{V}_{q}\right)$; расстояние между точками $\xi\left(\mathscr{P}_{0}, q^{\prime}\right)$ и $\xi\left(\mathscr{P}^{\prime}, q^{\prime}\right)$ в евклидовой метрике на $S^{2}$ меньше $\varepsilon$ для любого $q^{\prime} \in \mathbb{R}$. Существование такой окрестности $W$ следует из леммы 3. При этих условиях можно указать гомотопию

$$
H:[0,1] \times \mathbb{R} \rightarrow S^{2} \backslash\left(V_{p} \cup V_{q}\right),
$$

при которой точка $H\left(t, q^{\prime}\right)$ при изменении $t \in[0,1]$ пробегает однозначно определенную дугу (длины $<\pi)$ большого круга, содержащего точки $\xi\left(\mathscr{P}_{0}, q^{\prime}\right)$ и $\xi\left(\mathscr{P}^{\prime}, q^{\prime}\right)$ - концы этой дуги. Отсюда следует, что для любого $\mathscr{P}^{\prime} \in W$ диски $V_{p} \ni \xi\left(\mathscr{P}^{\prime}, p\right)$ и $V_{q} \ni \xi\left(\mathscr{P}^{\prime}, q\right)$ лежат в разных компонентах множества $S^{2} \backslash R_{\mathscr{P} \prime}[6$, теорема 2, с. 471$]$.

По лемме $1 e_{n-2} f^{-1}(T)$ связно и является объединением двух открытых множеств $U$ и $V$, причем $\mathscr{P} \in U \neq \varnothing$, следовательно, $V=\varnothing$ и $e_{n-2} f^{-1}(T)=U$. Отсюда следует, что множества (3) принадлежат разным компонентам множества $S^{2} \backslash R_{\mathscr{P} \prime}$ для любого $\mathscr{P}^{\prime} \in e_{n-2} f^{-1}(T)$.

При $y \neq A$ и $\mathscr{P}^{\prime \prime} \in e_{n-2} f^{-1}\left(\left[N^{\prime \prime}, E\right)\right)$ связное множество

$$
\xi\left(\mathscr{P}^{\prime \prime}, f^{-1}((D, E] \cup[E, A])\right)
$$

имеет общие точки с каждым из множеств (3) и, следовательно, с каждой из компонент $A_{\mathscr{P} \prime \prime}$ и $B_{\mathscr{P} \prime \prime}$, откуда следует, что оно имеет общие точки и с разделителем $R_{\mathscr{P} \prime \prime}$, однако это противоречит инъективности $\xi\left(\mathscr{P}^{\prime \prime}, \cdot\right)$. При $y=A$ и $\mathscr{P}^{\prime \prime} \in e_{n-2} f^{-1}\left(\left[L^{\prime}, E\right)\right)$ аналогичньми свойствами обладает множество

$$
\xi\left(\mathscr{P}^{\prime \prime}, f^{-1}((D, E] \cup[E, F])\right) .
$$

Таким образом, равенство (2) доказано.

Граф $K$ является $\Theta$-кривой. Положим $l_{0}=[A, B] \cup[A, C], l_{1}=[B, D] \cup[D, C]$, $l_{2}=[B, F] \cup[F, C]$. По лемме $2 Q_{K, \mathscr{P}}^{\prime}$ разделяет $S^{2}$ на три области $\Omega_{\mathscr{P}}^{0}, \Omega_{\mathscr{P}}^{1}$ и $\Omega_{\mathscr{P}}^{2}$ для любого $\mathscr{P} \in e_{n-2} f^{-1}(T)$, и в силу равенства $(2)$

$$
\xi\left(\mathscr{P}, f^{-1}(Y \backslash(K \cup G))\right) \subset \bigcup_{i=0}^{2} \Omega_{\mathscr{P}}^{i} .
$$

Используя леммы 1-3 и рассуждая как при доказательстве равенства (2), нетрудно показать, что для любого $\mathscr{P} \in e_{n-2} f^{-1}(T)$ множество $\xi\left(\mathscr{P}, f^{-1}(Y \backslash(K \cup G))\right)$ содержится в одной из этих компонент, скажем в $\Omega_{\mathscr{P}}^{1}$, причем по лемме 2

$$
\operatorname{Fr} \Omega_{\mathscr{P}}^{1} \subset \xi\left(\mathscr{P}, f^{-1}\left(l_{0} \cup l_{1}\right)\right) \text {. }
$$

Тогда

$$
\xi\left(\mathscr{P}, f^{-1}(F)\right) \cap \operatorname{Fr} \Omega_{\mathscr{P}}^{1}=\varnothing, \quad \mathscr{P} \in e_{n-2} f^{-1}(T) .
$$

Вместе с тем при $\mathscr{P}^{\prime} \in e_{n-2} f^{-1}\left(\left[L^{\prime}, E\right)\right)$ в силу леммы 3 связное множество

$$
\xi\left(\mathscr{P}^{\prime}, f^{-1}([E, D) \cup[E, F))\right)
$$

не пересекается с $\xi\left(\mathscr{P}^{\prime}, f^{-1}(K)\right)$, имеет общие точки с $\Omega_{\mathscr{P}}^{1}$ и, следовательно, содержится в $\Omega_{\mathscr{P}}^{1}$, однако это противоречит равенству (4).

На граф $K_{2}$ доказательство переносится с незначительными изменениями.

Как и в теореме 2 , под разбиением Мура компакта $Q$ понимаем разбиение $Q$ на компоненты множества $\bar{N}$ и отдельные точки $Q \backslash \bar{N}$. 
СлЕДСТВИЕ. Циклический континуум, являющийся пространством разбиения Мура компакта $Q$, допускающего $T(n, \mathbb{C})$-систему $(1)$ порядка $n>2$, гомеоморфен подмножеству сферы $S^{2}$.

Утверждение следует из теорем 2, 4 и теоремы Клэйтора [8] (см. также [6, замечание 2, с. 310$]$ ) о вложении в $S^{2}$ циклического локально связного континуума, не содержащего топологически графов $K_{1}$ и $K_{2}$.

\section{СПИСОК ЦИТИРОВАННОЙ ЛИТЕРАТУРЫ}

[1] Колмогоров А. Н. Замечание по поводу многочленов П. Л. Чебьшева, наименее уклоняющихся от заданной функции // УМН. 1948. Т. 3. №1. С. 216-221.

[2] Schoenberg I. J., Yang C. T. On the unicity of solutions of problems of best approximation // Ann. Mat. Pura Appl. 1961. V. 54. P. 1-12.

[3] Overdeck J. M. On the nonexistence of complex Haar systems // Bull. Amer. Math. Soc. 1971. V. 77. № 5. P. 737-740.

[4] Кошеев В. А. О чебышевских системах локально аналитических функций // Матем. заметки. 1994. Т. 55. № 3. С. 35-46.

[5] Федорчук В. В., Филиппов В.В.Общая топология. Основные конструкции. М.: Изд-во МГУ, 1988.

[6] Куратовский К. Топология. Т. 2. М.: Мир, 1969.

[7] Александров П. С. Топологические теоремы двойственности. Ч. 1. Замкнутые множества // Тр. МИАН. 1955. Т. 48. С. 5-108.

[8] Claytor S. Topological immersions of peanian continua in a spherical surface // Ann. of Math. 1934. V. 35. № 4. P. 809-835.

Институт математики и механики УрО РАН,

г. Екатеринбург 